\title{
La productividad de los autores en la ciencia de la información colombiana
}

\author{
Cristina Restrepo Arango \\ Maestría en bibliotecología el Colegio de México - México, \\ Distrito Federal. \\ E-mail: crestrepoarango@yahoo.com
}

\section{Rubén Urbizagástegui Alvarado}

Doctor en ciencia de la información. Universidad de California en Riverside. Biblioteca de Ciências - Riverside, CA 92521 5900, USA

E-mail: ruben@ucr.edu

\section{Resumen}

Este trabajo analiza la productividad científica de los autores que han publicado documentos en cuatro revistas de ciencia de la información editadas en Colombia desde 1978 a 2009. Se encontraron 555 autores, de los cuales 422 (76\%) publicaron un único artículo y 78 (24\%) publicaron 2 o más artículos. También se identificaron 11 autores como los más productivos con 21 y 7 artículos cada uno. El número de autores observados fueron estinados con los modelos del poder inverso generalizado, Gauss Poisson inversa generalizada y Poisson lognormal por el método de la máxima probabilidad. Las pruebas de ajuste estadístico chi-cuadrado y K-S mostraron que los tres modelos se ajustan a los datos observados en este trabajo.

\section{Palabras clave}

Ley de Lotka. Poder inverso generalizado. Gauss poisson inversa generalizada. Poisson lognormal. Bibliometría. Cienciometría. Informetría.

\section{Authors' productivity of colombian library and information science}

\author{
Abstract \\ The scientific productivity of the authors who have \\ published papers in four journals of library and information \\ science from Colombia since 1978 to 2009, was analyzed. \\ A total of 555 authors were found; 422 (76\%) of them \\ published a single article and 78 (24\%) published 2 or
}

more articles. Eleven authors were considered as the most productive with 21 and 7 articles respectively. The data was anal

The data was analyzed with the generalized inverse power, inverse generalized Gauss Poisson, and Poisson lognormal models by the maximum likelihood method. The statistical chi-square and $\mathrm{K}$-S tests showed that the three models fit the observed data.

\section{Keywords}

Lotka's law. Poisson lognormal. Generalized inverse power. Generalized inverse gauss poisson. Bibliometrics. Cienciometrics. Infometrics.

\section{INTRODUCCIÓN}

La investigación científica se inicia con la búsqueda de información, su consumo racional y la elaboración de un proyecto que finalizará con la difusión de sus resultados y hallazgos a través de la publicación de un documento. Este documento se diseminará a través de artículos publicados en revistas, capítulos de libros, ponencias presentadas en congresos $u$ otros tipos de documentos similares. La acumulación de las diversas formas de esos documentos ha dado lugar a los estudios sobre la productividad científica de los autores. Estos estudios comenzaron a principios del siglo XX con el trabajo de Dresden (1922) estudiando el campo de las matemáticas. Sin embargo, fue en 1926 que se propuso la ley del cuadrado inverso para medir la productividad de los autores en un campo científico (Lotka, 1926). Los estudios sobre la producción científica han permitido inferir que la producción es variable de acuerdo al campo de investigación, pero que se concentran en pocos autores, mientras que un gran número de ellos publican poco en su carrera académica. 
Esta preocupación por estudiar la productividad científica no sólo se ha centrado en el análisis del número de publicaciones, sino en los factores o variables que influyen directa o indirectamente en esa producción. Por ejemplo Fox (1983), hace una revisión de la literatura sobre este asunto y presenta una clasificación de las variables examinadas en diferentes estudios. Menciona que entre las variables se incluyen los rasgos psicológicos, hábitos de trabajo, características demográficas como la edad, contexto social y cultural y prestigio de la institución. Según estudios citados por esta autora, el prestigio de una institución puede ser un factor de peso para que un investigador incremente su producción, al igual que la edad en relación con la experiencia, la creatividad, los hábitos de trabajo, el capital cultural obtenido en la escuela y universidad, así como la libertad y flexibilidad para desarrollar proyectos de investigación. Al parecer también puede intervenir en la productividad el número de citas recibidas por los trabajos publicados, ya que estas puede ser un factor de motivación para continuar o no continuar con las publicaciones. Otro aspecto que puede influir en la productividad de un autor es la publicación de un determinado número de trabajos, pues, "es más probable que [un autor con una numerosa producción] escriba más artículos en el futuro que un autor menos prolífico" (Budd, 1988, p. 189).

La productividad científica también ha sido analizada desde la perspectiva psicológica a través del estudio de la creatividad de un investigador. Este tipo de estudios comenzó en 1835 y se han propuesto desarrollar modelos para medir la creatividad. Por ejemplo Simonton (1997), explica la productividad científica mediante el potencial inicial creativo. Considera como potencial creativo el número de ideas que surgen o están en desarrollo y el número de ideas convertidas en productos finales, es decir, en documentos científicos publicados. $\mathrm{Al}$ parecer la productividad es un fenómeno complejo que no se puede explicar con una única teoría, ya que son muchos los factores que intervienen en este proceso. Para Simonton (1997), sobre la productividad creativa intervienen afiliaciones profesionales, fluctuaciones económicas, conflictos militares y entre otros hasta enfermedades físicas.

También la productividad científica de los autores ha sido de interés para los sociólogos. La sociología busca una explicación de la productividad científica a través de la teoría de la ventaja acumulativa que está asociada con el Efecto Mateo propuesto por Robert Merton. El Efecto Mateo se basa en la frase bíblica: "pues al que tenga se le dará, y tendrá abundancia; pero al que no tenga se le quitará hasta lo poco que tenga". El Efecto Mateo describe "la acumulación del reconocimiento a las contribuciones científicas particulares de científicos de considerable reputación, y la negación de tal reconocimiento a los que todavía no se hayan distinguido". (Merton, 1977, p. 562). Por ello, Allison y Steward (1974, p. 597) sostienen que "la productividad está fuertemente asociada con el reconocimiento". Asimismo, la productividad científica es objeto de estudio de la cienciometría. Ésta busca dar respuesta a diferentes interrogantes tales como el comportamiento de la producción científica en determinada área del conocimiento, quiénes son los autores más productivos, cuál es el número de autores que sólo publican un único trabajo, cuál es la forma de crecimiento y obsolescencia de esa literatura, cuál es la estratificación de la productividad científica y cuál es la tasa de presencia de autores transeúntes, novatos, continuantes y prolíficos.

Este tipo de estudios son poco investigados en Colombia, especialmente en el campo de la bibliotecología y ciencia de la información. Por esa razón, el objetivo de este trabajo es analizar la productividad científica de los autores que han publicado documentos en cuatro revistas editadas en Colombia en las áreas de bibliotecología, archivística y ciencia de la información. El análisis se hará a través de la aplicación de la Ley de Lotka por los modelos del poder inverso generalizado, Gauss Poisson inversa generalizada y Poisson Lognormal, usando para los tres modelos el método de la máxima probabilidad. Estos modelos permitirán comparar cuál es el modelo que mejor predice la distribución de los datos observados 
La productividad de los autores en la ciencia de la información colombiana

sobre los autores que han publicado en estas revistas. Estas cuatro revistas son las que se han publicado en Colombia en los últimos 30 años y recogen lo más representativo de la producción nacional en este campo. Para lograr los objetivos propuestos, este trabajo está organizado en cuatro partes. Después de una somera introducción al asunto, la primera parte presenta una exhaustiva revisión de la literatura sobre los trabajos que se han realizado en el campo de BCI aplicando diferentes distribuciones estadísticas; la segunda parte describe la metodología que se usará en el desarrollo de este artículo; la tercera parte presenta los resultados encontrados, las conclusiones, y por último, la bibliografía usada en la elaboración de este artículo.

\section{REVISIÓN DE LITERATURA}

Como Lotka (1926) se limitó a estudiar la productividad científica de los autores en física y química, tal vez esa ley también se podría aplicar correctamente a las humanidades. Para probar este interés Murphy (1973), eligió la revista Technology and Culture especializada en historia de la tecnología. De esa revista seleccionó los artículos publicados a partir de enero de 1960 hasta diciembre de 1969. Encontró 170 autores que produjeron 231 artículos y verificó que la Ley de Lotka se aplicaba a la historia de la tecnología. Su método no está claramente detallado, pero utilizó el modelo del cuadrado inverso con $\mathrm{n}=2$ como el valor del exponente, en vez de calcular ese valor exponencial de los datos utilizados. Tampoco usó ninguna prueba estadística de la bondad del ajuste de los valores observados y esperados. Esos defectos hacen que el resultado de su estudio sea considerado poco fiable. Un año después Voos (1974), utilizó todos los artículos indizados en Information Science Abstract (ISA) de 1966 a 1970 y para cada año contó el número de autores que hicieron uno, dos, etc., contribuciones al campo de Bibliotecología y Ciencia de la Información (BCI) a estos datos aplicó la ley del cuadrado inverso. Consideró que la productividad en la Ciencia de la Información parecía ser de $1 / \mathrm{n}^{3.5}$, en lugar de $1 / \mathrm{n}^{2}$, conforme a lo sugerido por Lotka. También mostró que el 88\% de los autores escribieron sólo un artículo. Empleó el chi-cuadrado como prueba de la bondad del ajuste de los datos observados a los esperados. En ese mismo año Schorr (1974), examinó la aplicación de la ley de Lotka a las publicaciones que aparecieron en dos revistas de Bibliotecología: Library Quarterly (LQ) y College \& Research Libraries (C\&RL) para el período 1963-1972. Fueron estudiados un total de 618 contribuciones que incluían 210 artículos de LQ y 408 artículos de C\&RL. Encontró que la distribución de los datos se apartaba de la ley de Lotka y que en lugar de que los autores con dos artículos representasen el $25 \%$ de los autores individuales, estos representaban únicamente el $4,6 \%$ en cada revista. Concluyó que la ley de Lotka no se ajustaba al campo de la bibliotecología, debido a que las cuatro quintas partes de todos los documentos representaban la contribución de un único individuo. Sugirió que la ecuación de Lotka fuese alterada para ser de $1 / \mathrm{n}^{4}$ mediante la cual por cada 100 contribuyentes de un único artículo cerca de seis contribuirían con dos artículos, cerca de uno contribuirían con tres artículos, etc., es decir, sugirió la ley del cuádruple inverso.

Posteriormente Coile en una carta dirigida al editor de la revista Journal of the American Society for Information Science (1975), hizo comentarios sobre el artículo de Voos (1974) y realizó correcciones relacionadas con el número de autores, la ecuación usada y llamó la atención sobre el hecho de que los valores esperados no proporcionaban un ajuste adecuado a los datos. También llamó la atención sobre las diferencias entre los datos de Lotka y los datos de Voos relacionados con el tamaño de la muestra, períodos cubiertos y campo de aplicación. Ese mismo años Schorr (1975), aplicó el modelo del cuadrado inverso de Lotka a los datos publicados en una bibliografía sobre colecciones de mapas en el periodo de 1921 a 1973. Después de aplicar la prueba chi-cuadrado a sus datos, concluyó que la Ley de Lotka se ajustaba a este modelo. Dos años después Coile (1977), publicó un artículo refutando los resultados de Murphy (1973) y 
Schorr (1974) sobre los estudios de la ley de Lotka en las humanidades y sobre colecciones de mapas respectivamente. Para Coile los resultados encontrados por Murphy no se ajustaban al modelo del cuadro inverso por tres razones: la primera se refiere a que no utilizó la constante y el componente del cuadrado inverso del modelo propuesto por Lotka; la segunda porque que no usó el número total de autores como base de sus cálculos, sino el número real de autores únicos como base para el cálculo del número de autores esperados con dos artículos; la tercera porque sólo incluyó artículos académicos y también contó a los co-autores, mientras que Lotka sólo tuvo en cuenta el autor principal en el caso de artículos con más de dos autores. En relación al estudio de Schorr (1974), este autor encontró dos inconsistencias en los resultados: La primera se refiere a la aplicación del chi-cuadrado, debido a que no agrupó las contribuciones con valores inferiores a cinco; la segunda inconsistencia se refiere a que Shorr incluyó en su análisis a los coautores. Coile concluyó que los resultados de Shorr y Murphy no son empíricamente válidos debido a su desviación de la fórmula original de Lotka. También las pruebas estadísticas del chi-cuadrado y K-S rechazan el ajuste de los datos observados.

En la década de los ochenta Subramanyam (1981), discutió los resultados de Schorr (1974). Consideró que dos "incertidumbres" pueden haber causado el fracaso del ajuste del modelo del cuadrado inverso de Lotka: el limitado número de revistas seleccionadas y la falta de claridad en el conteo de algunos artículos con co-autorías. Este autor utilizó el volumen acumulado de 1978 de Library Literature y seleccionó sólo autores senior para colocar su estudio más en línea con la metodología de Lotka. Encontró que los patrones de autoría siguen estrechamente la Ley del cubo inverso en lugar del cuádruple inverso sugerido por Schorr. También observó que la proporción de los autores que contribuyeron con un sólo artículo es aproximadamente del 81\% del número total de los autores. Un año después Cline (1982), examinó los cambios que habían ocurrido en los patrones de publicación y citación de la revista C\&RL de 1939 a 1979. Encontró 1,240 autores principales que contribuyeron con 1,775 artículos publicados en este período con una media de 1,43 artículos por autor. Los datos son descritos en periodos agrupados de cinco a ocho años. Identificó y analizó los autores principales, el género, la afiliación institucional, la colaboración de autorías, las auto-citas, los documentos citados, la tasa de crecimiento de los documentos citados y los autores citados. Encontró que el 80\% (992) de los autores principales estudiados hicieron una única contribución a $C \& R L$. Una curva estimada de regresión lineal produjo $\mathrm{n}=2,44, \mathrm{C}=0,5929$ y N $=$ 1,240. Usando la prueba estadística KolmogorovSmirnov (K-S) al 0,01 nivel de significación concluyó que los datos empíricos no se ajustaban a Ley de Lotka, por lo tanto, era obvio que "los bibliotecarios no son tan productivos como los autores científicos" (Cline, 1982, p. 213). Claramente una conclusión errónea dada la limitación del estudio a una única revista cuando en el campo de la bibliotecología existían más de cien revistas.

En esta misma década Castillo Sánchez (1982), analizó la literatura sobre Bibliotecología difundida por diversas publicaciones seriadas hispanoamericanas disponibles en esa fecha en la biblioteca del Instituto Brasilero de Información Científica y Tecnológica (IBICT) en el Brasil. Identificó 878 artículos producidos por 53 revistas desde 1946 hasta 1979, es decir, un periodo de 33 años. Para fines de análisis redujo esa población a 606 artículos producidos por 47 revistas. Encontró que el $84 \%$ de los autores habían contribuido con un único artículo y que el 1,5\% de ellos proporcionaron entre 5 y 9 contribuciones. Concluyó que la Ley del cuadrado inverso de Lotka no se ajustaba a esta literatura. Cinco años después Foresti \& Martins (1987), analizaron los autores y artículos publicados desde 1980 hasta 1985 por cuatro revistas brasileñas de BCI. Observaron que 394 autores produjeron 559 artículos con $72 \%$ de estos autores publicando un único artículo y $0,27 \%$ de ellos publicando 10 artículos o más. Concluyeron que la Ley del cuadrado inverso de 
Lotka se aplicaba a este campo, pero no estimaron los valores esperados ni probaron estadísticamente la bondad del ajuste de los datos. Tsay (1989) analizó las revistas de indización y de resúmenes, con el fin de encontrar el núcleo de esas revistas, así como identificar la distribución de la productividad de los autores en esa área. Recolectó sus datos del "Indexing and abstracting: an international bibliography" editado por Wellisch cubriendo el período 1856 a 1976. En relación, con la ley de Lotka encontró que 1,966 autores produjeron 1,588 artículos y verificó que $78 \%$ de los autores habían contribuido con un único artículo. Usando el test estadístico de ajuste K-S concluyó que la productividad de autores se ajustaba bien al modelo del poder inverso generalizado de Lotka.

En la década de los noventa Budd y Seavey (1990), examinaron las tendencias de publicación de los bibliotecarios académicos para ver si su productividad se ajustaba a la ley de Lotka. Analizaron las autorías en 36 revistas de BCI en un período de cinco años, 1983-1987. El método del conteo fraccionado fue adoptado para los co-autores. Fueron revisados un total de 1,656 artículos escritos por 1,373 autores diferentes. Sólo 1,027 bibliotecarios tenían un artículo atribuido a su nombre y 128 individuos se identificaron como autores o coautores de más de dos artículos cada uno. Encontraron que los datos no se ajustaban al modelo del cuadrado inverso de Lotka. Vaishnav y Deo (1993) estudiaron el crecimiento de la literatura, las áreas temáticas cubiertas, la evolución de los asuntos y las tendencias de autorías usando una muestra de 25,669 resúmenes de la literatura sobre los servicios de bibliotecas e información computarizados recolectados en Library Information Science Abstracts (LISA) de los volúmenes 1970 y 1977-1990, pero solamente los volúmenes de los años 1970, 1978 y 1984-1989 (15,285 resúmenes) fueron considerados para un análisis detallado de la literatura midiendo el padrón de autoría, la productividad de los autores y la dispersión de la literatura. Encontraron que sólo $11,56 \%$ de la literatura fue el resultado de la colaboración, mientras que $88,44 \%$ fueron producidos por autores únicos. Observaron que el modelo del cuadrado inverso no se ajustó a los datos, pero si el modelo del poder inverso generalizado según la prueba estadística K-S. Sen, Taid y Hassa (1996) estudiaron la productividad científica en la bibliotecología y ciencia de la información indizada en LISA en el periodo de 1992 a 1993. Hallaron 7,624 autores en el año de 1992 y 6,423 autores en el año de 1993. Aplicaron la ley del cuadrado inverso de Lotka para los datos obtenidos en ambos años y compararon los resultados con diferentes valores de $\mathrm{n}$. Por ejemplo, con un valor de $\mathrm{n}=2$ y $\operatorname{con} \mathrm{n}=3,23$ para 1992 y $\operatorname{con} \mathrm{n}=3,1$ para 1993 y concluyeron que los valores estimados con un valor diferente a $\mathrm{n}=2$ se aproximaban más a los valores observados.

Al año siguiente Jiménez Contreras y Moya Anegón (1997), utilizando el modelo del poder inverso generalizado examinaron la producción científica de las revistas de biblioteconomía y documentación españolas en el periodo de 1975 a 1995. Encontraron 1,262 autores principales y coautores que produjeron 1,671 artículos con un promedio de 1,3 autores por artículo; el $70 \%$ de los autores produjeron un único trabajo. Los datos fueron probados con el test estadístico K-S con un nivel de significancia de 0,01 . Concluyeron que los datos se ajustaban al modelo estudiado con un valor crítico de 0,0459 y con una desviación máxima de 0,0259. Urbizagástegui Alvarado (1999) analizó el campo de la bibliometría para probar la aplicabilidad de la Ley de Lotka. Los datos fueron recolectados de "Bibliography of Bibliometrics and Citation Indexing \& Analysis" elaborado por Hjerppe y publicado en 1980. Esta bibliografía cubre un período de 80 años, tiempo suficiente para esperar que la productividad de los autores se aproxime a la distribución observada por Lotka en 1926. Adoptó el método del conteo directo, por lo tanto, sólo los autores principales fueron acreditados con la contribución de un artículo publicado. Analizó con el modelo del poder inverso generalizado 1,991 artículos producidos por 1,124 autores. Utilizando la prueba K-S a 
0,01 nivel de significancia encontró que esta literatura se ajusta a la distribución de Lotka.

Tres años después Bonnevie (2003), estudió la visibilidad, demografía de las autorías, internacionalización y comportamiento de las autocitas e impacto de la revista Journal of Information Science en el periodo de 1979 a 2001. En relación a los investigadores que publicaron en esa revista analizó 1,326 autores a través del modelo del poder inverso generalizado. Con el valor estimado de $\mathrm{n}=$ 2,618 y $\mathrm{C}=0,977$, encontró que los datos se ajustan a este modelo. Liu (2003) recolectó 208 artículos de investigación producidos por 364 autores del JASIS en el periodo de 2001 a 2000. Analizó la productividad de autores y las características de coautoría. De los 364 autores, 321 habían publicado un sólo un artículo representando $88,2 \%$ de la muestra. El patrón de distribución ideal fue estimado según el modelo del cuadrado inverso de Lotka. Los datos mostraron que la tasa observada de autores con dos o más artículos eran menores que los esperados de acuerdo a la ley de Lotka. Tsay (2004) aplicó el modelo del cuadrado inverso de Lotka a 10,238 autores que publicaron trabajos sobre indización temática en el periodo de 1977 a 2000 recuperados a través de LISA. Encontró que 1,847 (76,7\%) contribuyeron con un único trabajo con un promedio 1,4 artículos por autor. Los datos estimados fueron probados estadísticamente con el test K-S, mostrando que los datos no se ajustaban a este modelo.

En el mismo año Arquero Avilés y Salvador Oliván (2004), analizaron la procedencia institucional y la producción científica de los autores que contribuyeron con trabajos a las revistas y actas de congresos en el campo de la bibliotecología y documentación en español en el periodo de 1975 a 1984. Aplicaron la ley del cuadrado inverso de Lotka a 615 autores y encontraron que 497 autores contribuyeron con un sólo artículo, pero no incluyeron los datos estimados y tampoco usaron ninguna prueba estadística de ajuste de los datos, lo que compromete la validez de sus resultados. Asimismo, otros trabajos han aplicado el modelo de la ley del cubo inverso como Mittal y Singh (2006) que estudiaron la productividad científica y los aspectos relacionados con el crecimiento de la literatura, la identificación de las revistas más productivas y la distribución de artículos por países e idiomas sobre la literatura de educación bibliotecológica y ciencia de la información en el periodo de 1995 a 2004 y con datos recuperados de LISA. Aplicaron la ley del cubo inverso a los autores de 536 artículos, los cuales fueron divididos en dos grupos. Estos dos grupos fueron todos los autores del conteo completo y sólo los autores principales (conteo directo) para concluir que los datos estimados se alejan de los datos observados. Este artículo tampoco aplicó ninguna prueba estadística de ajuste a los datos, lo que compromete la validez de sus resultados.

En el mismo año Parreiras, Silva, Matheus y Brandão (2006), examinaron la colaboración a través del análisis de las redes sociales, así como la producción científica con la aplicación del cuadrado inverso de Lotka al campo de la BCI brasilera. Hallaron un valor de $\mathrm{n}=24,017$ y $\mathrm{C}$ $=0,7233 \mathrm{y}$ probaron el ajuste estadístico de los valores estimados con el test K-S que indicó que los datos se ajustaban a este modelo. Este trabajo no incluyó los valores observados ni los estimados. Debido al alto valor del exponente $\mathrm{n}$, tenemos serias dudas sobre la bondad del ajuste de estos resultados. Patra, Partha y Neera (2006) analizaron el padrón de crecimiento, el núcleo de las revistas y la distribución de los autores en el campo de bibliometría usando datos tomados de LISA desde sus inicios hasta el 2005. Aplicaron el modelo del cuadrado inverso y poder inverso generalizado de Lotka con la prueba de ajuste K-S y encontraron que la distribución de Lotka en ambos casos no se ajustaban a los dos modelos. Patra y Chand (2006) examinaron la productividad en bibliotecología y ciencia de la información en la India recuperados en la base de datos LISA en el periodo de 1967 a 2004. Encontraron 2,732 autores que publicaron 3,396 trabajos, cerca de 1,24 artículos por autor. De estos autores sólo 37 publicaron más de 10 artículos. Fueron analizados con el modelo del poder inverso 
La productividad de los autores en la ciencia de la información colombiana

generalizado de la ley de Lotka y probados con el test de K-S ajustándose a los datos observados. Yazid y Zainab (2007) analizaron las publicaciones producidas en Malasia en el campo de la BCI en el periodo de 1965 a 2005. Encontraron que 506 autores publicaron 1,045 publicaciones, de los cuales 309 (61\%) contribuyeron con un trabajo y 197 autores contribuyeron con dos y más trabajos. Aplicaron el modelo del cuadrado inverso de Lotka y concluyeron que esta ley se ajusta a los datos, porque el $60 \%$ de los autores contribuyeron con único trabajo. Sin embargo, no incluyeron los datos observados ni mencionan ningún test estadístico de ajuste de los datos, lo que pone en duda el ajuste de los datos a este modelo.

Posteriormente Gunasekera (2008), analizó la literatura sobre BCI en dos revistas editadas en Sri Lanka en el periodo de 1997 a 2007. Examinó los patrones de autoría, productividad de autores, patrones de citación, crecimiento, extensión de los artículos e idioma. Aplicó la ley del cuadrado inverso a 72 autores que contribuyeron con 141 artículos, de los cuales 45 contribuyeron con un artículo. A través de esa simple observación concluyó que los datos no se ajustan a este modelo, pues encontró que 63\% de los autores produjeron un sólo artículo. Este trabajo no incluye los datos estimados ni ninguna prueba de ajuste estadístico de los datos lo que compromete la fiabilidad de sus resultados. Urbizagástegui Alvarado (2008) utilizando la distribución Poisson Lognormal y Gauss Poisson inversa generalizada analizó la literatura estratificada producida sobre la ley de Lotka desde 1922 hasta 2003. Para este caso utilizó como formas de recuento de la literatura el conteo directo, conteo completo y conteo fraccionado. Los datos fueron evaluados con la prueba chi-cuadrado al 0,05 nivel de significación. Encontró que ambos modelos se ajustaban muy bien a la distribución de la literatura producida, pero el modelo Gauss Poisson generalizado inverso produjo un chi-cuadrado menor y predijo mejor el número total de autores que la distribución Poisson Lognormal.
Martín Sobrino; Pestana Caldes \& Pulgarín Guerrero (2008), estudiaron el campo de la ciencia de la información recolectando datos del Library and Information Science Abstracts (LISA), de 1996 al 2008. De esa base de datos recuperaron 2,825 registros correspondientes a 2,695 autores. Usando el método de conteo directo, el modelo del Poder inverso generalizado y la prueba K-S a un nivel de significancia de 0,01 , encontraron que los datos se ajustaban a la ley de Lotka. Askew (2008) analizó la literatura sobre BCI indizada en el Web of Knowledge. Aplicó el modelo del poder inverso generalizado a 2,109 autores que produjeron 1,856 artículos y halló un valor de $\mathrm{n}=2,1$ y un valor de $\mathrm{C}=$ 0,6418. Los datos estimados fueron evaluados con la prueba estadística K-S a un nivel de significancia de 0,10 con una desviación máxima de 0,022758 y un valor crítico de 0,026562l. Concluyó que los datos sobre la literatura en BCI se ajustaban a este modelo. Patra y Chand (2009) analizaron el crecimiento de la literatura, las principales revistas y los patrones de autoría en BCI producidos en el sur y sureste de Asia e indizados en LISA en el periodo de 1967 a 2005. Aplicaron el modelo del poder inverso generalizado a 3,260 autores que publicaron 3,958 artículos de los países del Sur de Asía y a 2,107 autores que publicaron 2,143 artículos de los países del Sur Este de Asia. Hallaron un valor de $\mathrm{c}=0,67 \mathrm{y} \mathrm{n}=-2,22$ para las publicaciones del sur de Asía y un valor de $\mathrm{c}=$ 0,72 y $n=-2,4$ para las publicaciones del sur este de Asia. Estos datos fueron probados con el test estadístico K-S que indicó que los datos del sur de Asia se ajustaron a este modelo, pero no así los datos del sur este de Asía.

En resumen, por la literatura revisada (Tabla 1) se puede observar que el modelo del cuadrado inverso no se ajusta a los datos observados cuando se usa un test estadístico para probar la bondad del ajuste de los datos. En general, el modelo del poder inverso generalizado parece ajustarse mejor a los datos observados igualmente los modelos Poisson Lognormal y Gauss Poisson Inversa Generalizada mencionados en esta revisión de la literatura. 
Cristina Restrepo Arango / Rubén Urbizagástegui Alvarado

TABLA 1

Artículos en BCI sobre la ley de Lotka desde 1973 hasta 2009

\begin{tabular}{|c|c|c|c|c|c|c|c|}
\hline Año & Autor & Modelo & Ajuste & Año & Autor & Modelo & Ajuste \\
\hline 1973 & Murphy & CI & No & 2003 & Bonnevie & PIG & Sí \\
\hline 1973 & Voos & CI & No & 2003 & Liu & CI & No \\
\hline 1974 & Schorr & CI & No & 2004 & Tsay & CI & No \\
\hline 1975 & Schorr & CI & No & 2004 & Arquero \& Salvador & $\mathrm{CI}$ & No \\
\hline 1977 & Coile & CI & No & 2006 & Mittal \& Singh & Cubo I. & No \\
\hline $\begin{array}{l}1981 \\
\text { (dudoso) }\end{array}$ & Subramanyan & CI & No & 2006 & Parreiras, et al. & CI & Sí \\
\hline 1982 & Cline & PIG & No & 2006 & Patra; et al. & $\mathrm{CI} / \mathrm{PIG}$ & No \\
\hline 1982 & Castillo & $\mathrm{CI}$ & No & 2006 & Patra \& Chand & PIG & Sí \\
\hline 1987 & Foresti \& Martins & $\mathrm{CI}$ & No & 2007 & Yazid \& Zainab & CI & No \\
\hline 1989 & Tsay & PIG & Sí & 2008 & Gunasekera & $\mathrm{CI}$ & No \\
\hline 1990 & Budd \& Seavy & $\mathrm{CI}$ & No & 2008 & Urbizagástegui & PL/GPIG & Sí \\
\hline 1993 & Vaishnav \& Deo & $\mathrm{CI} / \mathrm{PIG}$ & No /Sí & 2008 & Askew & PIG & Sí \\
\hline 1996 & Sen; Taid \& Hassa & $\mathrm{CI} / \mathrm{PIG}$ & No /Sí & 2008 & Martín; et al. & PIG & Sí \\
\hline 1997 & Jiménez \& Moya & PIG & Sí & 2009 & Patra \& Chand & PIG & Sí \\
\hline 1999 & Urbizagástegui & PIG & Sí & & & & \\
\hline
\end{tabular}

\section{METODOLOGÍA}

Las unidades de análisis fueron los autores que publicaron artículos en la Revista Interamericana de Bibliotecología (RIB), Revista de Ascolbi (RA), Revista Interamericana de Nuevas Tecnologías de la Información (RINTI) y Revista Códice (RC). Estos datos fueron obtenidos de 49 volúmenes y 124 fascículos publicados desde 1978 hasta 2009 por esas revistas. La RIB es una publicación académica editada semestralmente desde 1978 hasta la fecha por la Escuela Interamericana de Bibliotecología de la Universidad de Antioquia. La RA era una publicación de la Asociación Colombiana de Bibliotecólogos y Archivistas que se inició en 1988 y se dejó de publicar a partir del año de 1992. La RINTI editada por el Departamento de Ciencias de la Información de la Pontificia Universidad Javeriana se publicó desde el año 1995 hasta el 2003. La RC es una publicación de la Facultad de Sistemas de Información y Documentación de la Universidad de la Salle se edita desde el año 2005 hasta la fecha.

La RIB y la RC son las dos únicas revistas que continúan editándose en Colombia en el campo de la bibliotecología, archivística y ciencia de la información (BACI), siendo la más antigua y productiva la RIB. Las revistas RINTI y RA no se publican desde el año 2003 y 1992 respectivamente, aunque la RA sólo se editó durante cinco años era la segunda revista con el mayor número de artículos sobre BACI. La RINTI es la única de estas cuatro revistas que además de publicar artículos sobre BACI en Colombia también difundió artículos relacionados con las tecnologías de la comunicación y otros campos del conocimiento. Estas cuatro revistas cubren el espectro de autores que publican en Colombia desde 1978 hasta diciembre del 2009.

Los autores que han difundido sus trabajos a través de estas revistas se recogieron directamente de las tablas de contenido disponibles en las páginas Web de estas revistas. Los datos que no se encontraron en las páginas Web de las revistas se obtuvieron a través de la consulta de las analíticas de revistas del catálogo electrónico del Sistema de Biblioteca de la Universidad de Antioquia y/o a través de su servicio de referencia virtual. De la RINTI sólo se tuvieron en cuenta los artículos sobre BACI, pero no se incluyeron los datos del volumen 8 del año 2003, porque no fue posible para los autores de 
este trabajo acceder al número 1 a pesar del envío de solicitudes al editor de la revista.

Los datos pertinentes para el análisis fueron organizados en una hoja de cálculo en Open Office que incluye los campos de título del artículo; volumen, número y año, así como los autores principales y coautores de los artículos. De estos datos se extrajeron los nombres y apellidos de los autores y el número de contribuciones de cada uno de ellos. El método de conteo empleado fue el conteo completo, es decir, se consideró como autores tanto a los autores principales como a los coautores.

Como el modelo del cuadrado inverso no soporta la prueba del chi-cuadrado o K-S, ahora es conocida como la ley de Lotka una forma más general llamada poder inverso generalizado que se expresa como:

$\mathrm{y}_{\mathrm{x}}=\mathrm{kx}^{-\mathrm{b}}, \quad \mathrm{x}=1,2, \ldots, \mathrm{x}_{\max }$

donde,

$\mathrm{y}_{\mathrm{x}}$ es la probabilidad de que un autor haga $\mathrm{x}$ contribuciones sobre un asunto

$\mathrm{k}$ y b son los dos parámetros que deben ser estimados de los datos observados.

Para estimar el parámetro k se usó la función Zeta inversa de Riemann, para el que Pao (1985) proporciona una fórmula de aproximación exacta. La estimación del parámetro b se hizo a través del método de la máxima probabilidad y usando los valores de la tabla proporcionada por Johnson \& Kotz (1969). Esta Tabla ha sido recalculada y reproducida tanto por Rousseau (1993) como por Nicholls (1988). Los valores esperados fueron estimados usando el software NLREG (NonLinear Regression).

La distribución Poisson Lognormal fue propuesta por Steward (1994). Según este modelo, los datos recolectados para el análisis de la productividad de los autores generalmente producen una forma J inversa cero truncada, con una larga cola de grandes productores. Eso hace que el modelo Poisson Lognormal sea un candidato ideal para probar este tipo de distribuciones discretas. El modelo es descrito como una distribución compuesta, donde la propensión subyacente d de los científicos para publicar un artículo sigue una distribución Lognormal. Dado la propensión subyacente d específica de un científico, su probabilidad Px de publicar x artículos, sigue un simple modelo Poisson:

$$
P_{x}=\frac{\delta^{x} e^{-\delta}}{x !} \quad \text { para } \quad x=0,1,2,3, \ldots
$$

Siendo así, la distribución de los valores observados de todos los autores que tengan el mismo valor d tendrán una distribución con una media y una varianza d. En una muestra de autores cuyos logaritmos d están normalmente distribuidos con una media $\mathrm{m}$ y un desvío padrón $\mathrm{s}$, las Px de la muestra total son proporcionadas por la siguiente ecuación:

$P_{x}=\frac{1}{\sigma \sqrt{2 \pi}} \frac{1}{x !} \int_{o}^{\infty} e^{-\delta} \delta^{x-1} \exp \left\{-\frac{(\ln \delta-\mu)^{2}}{2 \sigma^{2}}\right\} d \delta$

para $\mathrm{x}=0,1,2,3, \ldots \ldots$

Los valores esperados de este modelo Poison Lognormal fueron estimados con la ayuda de un software gratuitamente proporcionado por el Profesor John A. Steward (2005).

La distribución Gauss Poisson inversa generalizada fue desarrollada por Sichel (1982, 1985, 1986, 1992) y ahora este modelo es conocido como la "distribución de Sichel". Este modelo de tres parámetros puede ser representado como:

$\phi(r \mid t)=\frac{\left(1-t_{t}\right)^{\frac{y}{2}}}{K_{y}\left\{\alpha_{t}\left(1-\theta_{t}\right)^{\frac{1}{2}}\right\}} \frac{\left(\frac{\alpha_{t} \theta_{t}}{2}\right)^{r}}{r !} K_{r+y}\left(\alpha_{t}\right)$

donde,

y 
Las variaciones y características de dependencia del tiempo y otras propiedades de este modelo son proporcionadas por Sichel (1982). Las probabilidades individuales se obtienen de la ecuación de recurrencia general,

$\hat{\phi}(r)=\left(\frac{r+\gamma-1}{r}\right) \theta_{t} \phi(r-1)+\frac{\alpha_{t}^{2} \theta_{t}^{2}}{4 r(r-1)}$

Esta ecuación puede ser simplificada haciendo que $g$ tenga un valor de medio integral. Entonces si se supone que $\gamma=-\frac{1}{2}$ a priori, entonces la distribución discreta es,

$\phi(r)=\left(\frac{2 \alpha}{\pi}\right)^{\frac{1}{2}} \exp \left\{\alpha(1-\theta)^{\frac{1}{2}}\right\} \frac{\left(\frac{\alpha \theta}{2}\right)^{r}}{r !} K_{r-}$

Las pruebas de ajuste usadas para los tres modelos estudiados son chi-cuadrado (a un nivel de significancia 0,05 ) y K-S (a un nivel de significancia de 0,01). La prueba K-S compara la función de densidad de los valores observados con la de los valores esperados o calculados. Una de sus ventajas es que trabaja muy bien con pequeñas muestras, no pierde información con la agrupación de los datos en clases y es más poderosa que el chi-cuadrado. Para la aplicación de la prueba del chi-cuadrado ninguno de los valores de las frecuencias observadas deben ser inferiores a cinco, por lo tanto, las células observadas con autores menores a 5 fueron acumulados con las células adyacentes para producir autores agrupados iguales o mayores a 5 .

\section{RESULTADOS}

La Figura 1 es la representación gráfica del número de contribuciones según el número de autores encontrados en esta investigación; 422 (76\%) autores han publicado un único artículo, mientras que $133(24 \%)$ autores han publicado 2 o más artículos cada uno. Esto significa que la BACI colombiana está dominada por autores transeúntes y que solamente el $24 \%$ de la población total de productores persisten con nuevas publicaciones.

\section{FIGURA 1}

\section{Contribuciones según el número de autores}

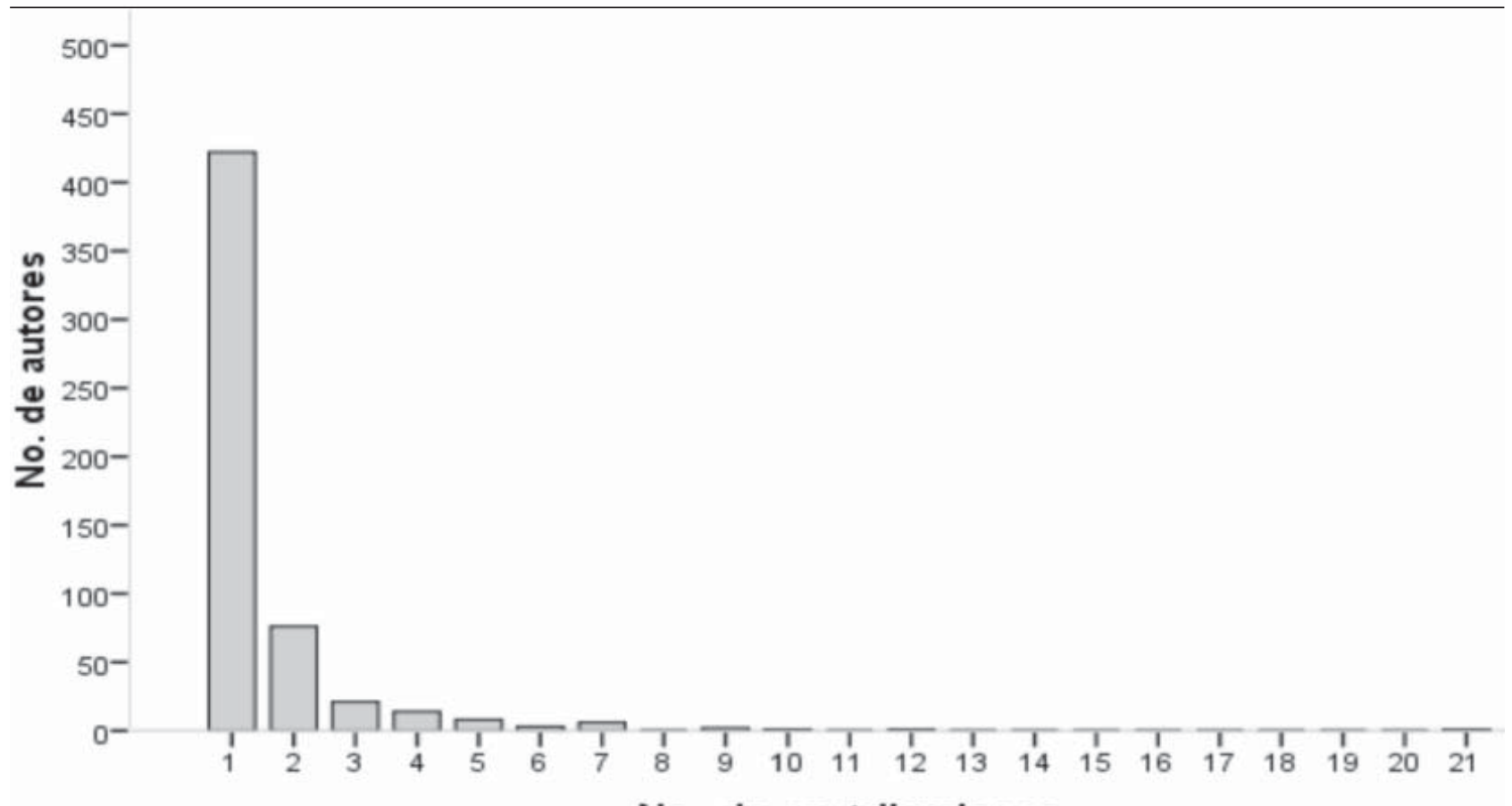

\section{No. de contribuciones}


De este $24 \%$ apenas $3(0,5 \%)$ autores pueden ser considerados como persistentes. La producción media de los autores fue de 1,5 artículos con una varianza de 2,3 y un índice de dispersión de 1,53 artículos. Los autores únicos observados son $16 \%$ más altos que los encontrados por Lotka (1926) en el campo de la física y la química.

La Tabla No. 2 presenta el número de autores observados y estimados de acuerdo a los modelos estudiados a través del método de la máxima probabilidad. En relación al total de los autores (555), el modelo del poder inverso generalizado (PIG) estima $+5,34$ autores, el Gauss Poisson inversa generalizada (GPIG) estima $+2,52$ autores y el Poisson Lognormal (PL) estima -0,51 autores. El modelo PL es el que estima más cercanamente el valor total de los autores observados seguido del modelo GPIG y finalmente el modelo PIG. Igualmente si observamos cada una de las filas estimadas de las contribuciones los valores más cercanos a los valores observados son proporcionados por los mismos modelos y en el mismo orden.

\section{TABLA 2}

\section{Autores estimados según los modelos}

\begin{tabular}{|c|c|c|c|c|}
\hline $\begin{array}{l}\text { Contri- } \\
\text { buciones } \\
\text { X }\end{array}$ & $\begin{array}{c}\text { Autores } \\
\text { observados } \\
\text { Y }\end{array}$ & $\begin{array}{c}\text { Autores } \\
\text { estimados } \\
\text { PIG }\end{array}$ & $\begin{array}{c}\text { Autores } \\
\text { estimados } \\
\text { GPIG }\end{array}$ & $\begin{array}{c}\text { Autores } \\
\text { estimados } \\
\text { PLN }\end{array}$ \\
\hline 1 & 422 & 422.35 & 422.02 & 420.57 \\
\hline 2 & 76 & 73.56 & 70.64 & 77.73 \\
\hline 3 & 21 & 23.47 & 27.27 & 25.81 \\
\hline 4 & 14 & 12.82 & 13.51 & 11.55 \\
\hline 5 & 8 & 7.30 & 7.58 & 6.44 \\
\hline 6 & 3 & 4.61 & 5.50 & 3.65 \\
\hline 7 & 6 & 3.13 & 3.50 & 2.34 \\
\hline 8 & 0 & 2.23 & 2.30 & 1.59 \\
\hline 9 & 2 & 1.66 & 1.56 & 1.13 \\
\hline 10 & 1 & 1.27 & 1.87 & 0.83 \\
\hline 11 & 0 & 1.00 & 0.76 & 0.62 \\
\hline 12 & 1 & 0.80 & 0.54 & 0.48 \\
\hline 13 & 0 & 0.66 & 0.39 & 0.38 \\
\hline 14 & 0 & 0.54 & 0.28 & 0.30 \\
\hline 15 & 0 & 0.46 & 0.20 & 0.25 \\
\hline 16 & 0 & 0.39 & 0.14 & 0.20 \\
\hline 17 & 0 & 0.33 & 0.10 & 0.17 \\
\hline 18 & 0 & 0.29 & 0.07 & 0.14 \\
\hline 19 & 0 & 0.25 & 0.05 & 0.12 \\
\hline 20 & 0 & 0.22 & 0.03 & 0.10 \\
\hline 21 & 1 & 0.19 & 0.02 & 0.09 \\
\hline Total & 555 & 560.34 & 557.52 & 554.49 \\
\hline
\end{tabular}

En la Tabla No. 3 se presentan los valores estimados y críticos de los test estadísticos chi-cuadrado y K-S utilizados para probar la bondad del ajuste de los modelos a los datos analizados en este trabajo. Los tres modelos se ajustan estadísticamente a los datos de la productividad de los autores que publicaron sus trabajos en las cuatro revistas colombianas de BACI. El modelo que mejor predice los valores observados es el Poisson Lognormal, ya que muestra un menor chi-cuadrado y K-S, le sigue el modelo Gauss Poisson inverso generalizado y finalmente el poder inverso generalizado.

TABLA 3

Valores estimados y críticos de las pruebas estadísticas

\begin{tabular}{lcccc}
\hline Modelos & \multicolumn{2}{c}{ Chi-Cuadrado } & \multicolumn{2}{c}{ Kolmogorov-Smirnov } \\
& Estimado & Valor Crítico & Dmx & Valor crítico \\
\hline PIG & 2,6568 & 11,0705 & 0,0121 & 0,0692 \\
GPIG & 2,3623 & 7.81473 & 0,0128 & 0,0692 \\
PL & 2,1109 & 9,48773 & 0,06873 & 0,0692 \\
\hline
\end{tabular}

La Tabla 4 presenta los autores con el mayor número de contribuciones en las revistas de BACI en Colombia. Los tres autores más productivos fueron José Arias Ordoñez (21 artículos), Bertha Nelly Cardona Rave (12 artículos) y Didier Álvarez Zapata (10 artículos) y los 8 restantes publicaron entre 7 y 9 trabajos, es decir, el $2 \%$ de los autores son responsables del 10,7\% de la producción total de artículos en Colombia.

TABLA 4

Autores más productivos en la BACI en Colombia

\begin{tabular}{|c|c|c|c|c|c|}
\hline \multirow{2}{*}{$\begin{array}{l}\text { Nombre de } \\
\text { los autores }\end{array}$} & \multicolumn{4}{|c|}{ Publicados en las revistas } & \multirow{2}{*}{$\begin{array}{l}\text { Total de } \\
\text { artículos }\end{array}$} \\
\hline & RIB & RA & RINTI & RC & \\
\hline Arias Ordoñez, José & 8 & 2 & 11 & -- & 21 \\
\hline Cardona Rave, Bertha Nelly & 10 & 2 & - & -- & 12 \\
\hline Álvarez Zapata, Didier & 10 & -- & - & -- & 10 \\
\hline Herrera Cortés, Rocío & 9 & -- & -- & -- & 9 \\
\hline Jaramillo, Orlanda & 9 & - & -- & -- & 9 \\
\hline Arboleda Sepúlveda, Orlando & 6 & -- & -- & 1 & 7 \\
\hline Delgado Torres, Nora Alicia & 7 & -- & -- & -- & 7 \\
\hline Moncada Patiño, José Daniel & 7 & - & - & -- & 7 \\
\hline Múnera Torres, María Teresa & 7 & - & - & -- & 7 \\
\hline Rendón Giraldo, Nora Helena & 7 & -- & -- & -- & 7 \\
\hline Yepes Osorio, Luis Bernardo & 6 & -- & 1 & -- & 7 \\
\hline
\end{tabular}


Sobre esta concentración de publicaciones de pocos autores en una misma revista, Bonnevie (2003, p.16), cita la "teoría de la concentración y el equilibrio" propuesto por Reiter, Steiner y Werner (1977) que afirma que en los años iniciales de una revista se percibe una concentración relativamente alta de autores que publican constantemente en esa revista. Esta constancia disminuye paulatinamente para dar oportunidad a los nuevos contribuyentes, hasta que finalmente se llega a un equilibrio; pero que las revistas no exitosas no disminuyen su concentración en la misma medida hasta que finalmente, a veces, dejan de ser publicadas. Una posible explicación de este proceso es que al principio, un grupo de autores que han organizado la publicación de la revista dominan, acaparan y concentran las publicaciones (hipótesis de concentración). Sin embargo, con el pasar del tiempo, la revista alcanza un nivel de normalización que es indicado por el hecho de que muchos nuevos investigadores someten a la revista cada vez más documentos para su publicación. Por lo tanto, disminuye la concentración de publicaciones de los autores iniciales y/o ligados a la revista y se alcanza así un punto de equilibrio, que indica la madurez de la revista (hipótesis de equilibrio).

Esto no parece ser el caso de la RIB ni de la RINTI en las que se observa alta concentración de autores publicando en la misma revista en la que también son editores o parte del entorno editorial de las revistas. Quizá, esto se deba a la falta de oportunidades para publicar en otras revistas existentes en el país o a la falta de osadía para someter artículos a revistas existentes en la región. También sospechamos que en parte se debe a la carencia de cursos de postgraduación y por ende a la falta de fortalecimiento de las capacidades de dominio de las metodologías de investigación científica que influye en la calidad de los resultados de investigación en el área en Colombia. Por ejemplo Patra y Chand (2009), encontraron que los países de Asia con mayor producción científica en BCI indizada en la base de datos LISA eran los que contaban con cursos de post-graduación en esa región. Este es el caso de la India. Esta misma tendencia fue encontrada por Gunasekera (2008) en la producción científica en BCI en Sri Lanka.

Asimismo, estos autores difunden sus artículos preferentemente en las revistas editadas por las instituciones a las cuales están afiliados. De los 11 autores más productivos 7 autores sólo han publicado a través de la RIB, de los cuales 6 fueron o han sido docentes o directores de la institución responsable de la edición de la revista. Igualmente, el más productivo de los 11 autores publicó 11 artículos en la RINTI en la cual figura como editor. Sólo 2 de los autores más productivos no laboran en esas instituciones colombianas.

Esta preferencia de publicar en la propia revista donde se es parte del consejo editorial es denominada en la literatura como endogamia académica. Este término es definido por Fernández Argüelles (2004, p. 3) como la práctica que incluye "todas las actitudes y acciones tendientes a formar grupos (de docentes o investigadores, fijos o circunstanciales, aislados o abarcadores) entre personas relacionadas, directa o indirectamente, por vínculos que los comprometen con el grupo, ya sean estos de naturaleza familiar, laboral o social. De esta manera se mezclan los intereses académicos con los personales ocasionando conflictos que provocan homogeneidad y debilidad del arbitraje o control que conspira contra la calidad de los procesos académicos". Se entiende entonces que cuando se trata de la publicación de artículos académicos la marcada tendencia a publicar en las revistas donde se es o parte del comité editorial o parte del cuerpo académico responsable de la edición de la revista estamos hablando de una práctica endogámica. De hecho, la endogamia favorece la productividad cuantitativa de los comprometidos en esa práctica, pero compromete también la credibilidad de la calidad de lo publicado, pues, lanza dudas respecto a si merecieron ser publicados o si fueron publicados solamente para reforzar los intereses académicos y personales ocasionando al mismo tiempo la debilidad del arbitraje de la revista. Por lo tanto, cabe hablar de endogamia "cuando los miembros de los comités editoriales pueden utilizar 
La productividad de los autores en la ciencia de la información colombiana

sus revistas para publicar preferentemente trabajos propios o trabajos de miembros de sus equipos de investigación” (Fernández Argüelles, 2004).

\section{CONCLUSIONES}

En Colombia en el periodo estudiado de 1978 a 2009 se encontraron 555 autores que publicaron sus trabajos a través de cuatro revistas, de los cuales el $76 \%$ publicaron un solo artículo siendo estos considerados como autores transeúntes y $24 \%$ de los autores publicaron dos o más artículos que los posicionan como autores continuantes. De este total sólo 11 son los más productivos, los cuales contribuyeron entre 21 y 7 artículos. Estos datos se ajustan al modelo de Lotka, aunque el modelo que mejor predijo los datos observados fue Poisson lognormal, lo cual fue confirmado por los resultados de las pruebas estadísticas chi-cuadrado y K-S. En orden de magnitud del ajuste le sigue el modelo Gauss Poisson inverso generalizado y finalmente el poder inverso generalizado.

Incidentalmente también observamos que en la BACI colombiana hay una práctica endogámica en la publicación de artículos en sus revistas, ya que los autores más productivos identificados en esta investigación estuvieron o están vinculados institucionalmente al comité editorial de las revistas. Sería conveniente establecer políticas y criterios de evaluación que corrijan estas prácticas y que motiven a la difusión de los resultados de investigaciones en revistas diferentes a las de la propia institución. Asimismo, los editores deben desarrollar estrategias de difusión, con el fin de recibir artículos de académicos de otras instituciones y países. Estas estrategias y políticas incrementarían la visibilidad de las revistas y los investigadores.

Artigo submetido em 09/11/2010 e aceito em 08/11/2011.

\section{BIBLIOGRAFÍA}

ALLISON, Paul D.y STEWARD, John A. Productivity differences among scientists: evidence for accumulative advantage. American sociological review, v. 39, n. 4, p. 596-606, 1974.

ASKEW, Consuella Antoinette. An examination of Lotka's law in the field of Library and Information Studies. Miami, FL: Florida International University (Tesis para optar al de Doctorado en Educación), 2008. Disponible en: <http://digitalcommons.fiu.edu/cgi/viewcontent. coiparticle $=1235 \&$ context $=$ etd $>$. Acceso en: 13 Sep. 2010 .

ARQUERO AVILÉS, Rosario y OLIVÁN, José Antonio Salvador. Evaluación y análisis de la producción de los autores de trabajos difundidos en publicaciones periódicas editadas en español: área de Biblioteconomía Documentación 1975-1984. Documentación de las Ciencias de la Información, v. 27, p. 53-74, 2004.

BONNEVIE, Ellen. Science a multifaceted portrait of a library and information science journal: the case of the Journal of Information Science. Journal of Information Science, v. 29, n. 1, p. 11-23, 2003.

BUDD, John M. y SEAVEY, Charles A. Characteristics of journal authorship by academic librarians. College \& Research Libraries, v. 51, n. 5, p. 463-470, 1990.

BUDD, John M. A bibliometric analysis of higher education literature author(s). Research in Higher Education, v. 28, n. 2, p. 180190, 1988.

DRESDEN, Arnold. A report on the scientific work of the Chicago Section, 1897-1922. Bulletin of The American Mathematical Society, v. 28, p. 303-307, 1922. Disponible en: < http://projecteuclid.org/ DPubS/Repository/1.0/Disseminate?view=body\&id=pdf_1\&han dle=euclid.bams/1183485109>. Acceso en: 31 Ago. 2010.

CASTILLO SÁNCHEZ, Octavio. Aplicación de la Ley de Lotka a la literatura bibliotecologica hispanoamericana existente en la Biblioteca del Instituto Brasileiro de Informaçăo em Cięncia e Tecnologia (IBICT), Rio de Janeiro, Brasil, Boletín del Departamento de Bibliotecología de la Universidad de Panamá, v. 6, p. 17-24, 1982.

CLINE, Gloria S. College \& Research Libraries: its first forty years, College \& Research Libraries, v. 43, n.3, p. 208-232, 1982.

COILE, Russell C. Lotka's frequency distribution of scientific productivity, Journal of the American Society for Information Science, v. 28, n. 6, p. 366-370, 1977.

COILE, Russell C. Lotka and information science, Journal of the American Society for Information Science, v. 26, n. 2, p. 133, 1975.

FERNÁNDEZ ARGÜELLES, Rogelio. La endogamia académica universitaria: una visión desde el siglo XX". En Cuarto Congreso Nacional y Tercero Internacional: "Retos y Expectativas de la Universidad" Ejes: Desarrollo Universitario - Desarrollo de Actores y Participantes Sede: Universidad Autónoma de Coahuila Febrero 25-28, 2009. Disponible en: < http://www.congresoretosyexpectativas.udg.mx/ Congreso\%204/Mesa\%203/m302.pdf>. Acceso en: 16 sep. 2010.

FORESTI, Noris Almeida Betónico y MARTINS, Maria Sueli Machado. Revistas brasileiras de Biblioteconomia, Documentaçăo e Cięncia da Informaçăo: produtividade de autores no período de 1980 a 1985. Revista da Escola de Biblioteconomía da Universidade Federal de Minas Gerais, v. 16, n. 1, p. 54-71, 1987. 


\section{Cristina Restrepo Arango / Rubén Urbizagástegui Alvarado}

FOX, Mary Frank. Publication productivity among scientist: a critical review, Social Studies of Science, v. 13, n. 2, p. 285-305, 1983.

JIMÉNEZ CONTRERAS, Evaristo y MOYA ANEGÓN, Félix de. Análisis de la autoría en revistas españolas de biblioteconomía y documentación, 1975-1995, Revista Española de Documentación Cientifica, vol. 30, no. 3, p. 252-266, 1997.

JOHNSON, Norman Lloyd y KOTZ, Samuel. Discrete distributions. Boston: Houghton Mifflin [1969].

GUNASEKERA, Chamani. Library and information sciences research literature in Sri Lanka: a bibliometric study. Journal of the University Librarians Association of Sri Lanka. v. 12, p. 17-46, 2008.

LIU, Jingjing. A bibliometric study: author productivity and coauthorship features of JASIST 2001-2002, Mississippi Libraries, v. 67, n. 4, p. 110-112, 2003.

LOTKA, Alfred. The frequency distribution of scientific productivity. Journal of the Washington Academy of Sciences, v. 16, n. 12, p. 317-323, 1926. Traducción de Rubén Urbzagástegui Alvarado.

MARTÍN SOBRINO, Maria Isabel; PESTANA CALDES, Ana Isabel \& PULGARÍN GUERRERO, António. Lei de Lotka aplicada à produção científica da área de ciência da informação. Brazilian Journal of Information Science, v. 2, n. 1, p. 16-32, jan./jun., 2008. Disponível em: <http://www.bjis.unesp.br/>.

MERTON, Robert K. La sociología de la ciencia, 2: investigaciones teóricas y empiricas. Recopilación e introducción de Norman W. Storer; Versión española de Néstor Alberto Míguez. Madrid: Alianza Editorial, 1977, v. 2.

MITTAL, Rehka \& SINGH, Gian. Periodical literature on Library and Information science educaction: a bibliometric study. Annals of Library and Information Studies, v. 53, p. 224-229, 2006.

MURPHY, Larry J. Lotka's law in the Humanities? Journal of the American Society for Information Science, v. 24, n. 6, p. 461-462, Nov.-Dec., 1973.

NICHOLLS, Paul Travis. Price's square root law: empirical validity and relation to Lotka's law. Information Processing Management, v. 24, n. 4, p. 469-477, 1988.

PAO, Miranda Lee. An empirical examination of Lotka's Law. Journal of the American Society for Information Science, v. 37, n. 1, p. 26-33, 1986.

PAO, Miranda Lee. Lotka's law: a testing procedure. Information Processing \& Management, v. 21, n. 4, p. 305-320, 1985.

PATRA, Swapan Kumar \& PRAKASH, Chand. Library and information science research in SAARC and ASEAN contries and reflected throught. Annals of Library and Information Studies, v. 56, p. 41-51, 2009.

PATRA, Swapan Kumar y PRAKASH, Chand. Library and information science research in India: A bibliometric study. Annals of Library and Information Studies, v. 53, p. 219-223, 2006.

PATRA, Swapan Kumar; BHATTACHARYA, Partha \& VERMA, Neera. Bibliometric Study of Literature on Bibliometrics. DESIDOC Bulletin of Information Technology, v. 26, n. 1, p. 2732, Jan., 2006.

PARREIRAS, Fernando Silva; SILVA, Antonio Braz de Oliveira e; MATHEUS, Renato Fabiano y BRANDÃO, Wladmir Cardoso.
REDECI: colaboração e produção científica em ciência da informação no Brasil. Perspectivas em Ciencia da Informacao, v. 11, n.3, p. 1-16, 2006.

ROUSSEAU, Ronald. A table for estimating the exponent in Lotka's law. Journal of Documentation. v. 49, n.4, p. 409-412, 1993.

SCHORR, Alan Edward. Lotka's law and library science. RQ [Reference Quarterly], v. 14, n. 1, p. 32-33, 1974.

SCHORR, Alan Edward. Lotka's law and map librarianship. Journal of the American Society for Information Science, v. 26, n. 3, p.189-190, 1975.

SEN, B. K., TAID, Che Azlan bin y HASSAN, Mohd Faris Bin. Library and information science literature and Lotka's Law. Malasyan Journal of Information Science, v. 1, n. 2, p. 89-93, 1996.

STEWARD, John A. Comunicación personal, 2005.

STEWARD, John A. The Poisson Lognormal model for bibliometric/scientometrics distributions. Information Processing Management, v. 30, n. 2, p. 239-251, 1994.

SICHEL, H. S. Anatomy of the generalized inverse GaussianPoisson distribution with special applications to bibliometric studies. Information Processing and Management, v. 28, n. 1, p. 5-17, 1992.

SICHEL, H. S. The GIGP distribution model with applications to physics literature. Czechoslovak Journal of Physics, v. B36, n. 1, p. 133137, 1986.

SICHEL, H. S. A bibliometric distribution which really works. Journal of the American Society for Information Science, v. 36, n. 5, p. 314-321, 1985.

SICHEL, H. S. Asymtotic efficiencies of the three methods of estimation for the inverse Gaussian Poisson distribution. Biometrika, v. 69 , p. $467-472,1982$.

SUBRAMANYAM, Krishna. Lotka's Law and library literature. Library Research, v. 3, n. 2, p. 167-170, 1981.

TSAY, Ming-Yueh. Literature growth, journal characteristics, and author productivity in subject indexing, 1977 to 2000 . Journal of the American Society for Information Science and Technology, v. 55 , n. 1, p. 64-71, 2004.

TSAY, Ming-Yueh. A bibliometric study of indexing and abstracting, 1876-1976. Indexer, v. 16, n. 4, p. 234-238, Oct., 1989.

URBIZAGÁSTEGUI ALVARADO, Rubén. A produtividade dos autores sobre a lei de Lotka. Ciência da Informação, v. 37, n. 2, p. 87-102, Maio-Ago, 2008.

URBIZAGÁSTEGUI ALVARADO, Rubén. La ley de Lotka y la literatura de Bibliometría. Investigación Bibliotecológica, v. 13, n. 2, p. 125-141, July-Dec., 1999.

VAISHNAY, A. A. y DEO, V. N. Literature of computerised library and information services: a bibliometric study Library. Science with a Slant to Documentation and Information Studies, v. 30, n. 2, p. 45-53, June, 1993.

VOOS, Henry. Lotka and Information Science. Journal of the American Society for Information Science, v. 25, n. 4, p. 270-272, 1974.

YAZID, Norhazwani y ZAINAD, A. N. Publication productivity of Malaysian authors and Institutions in LIS. Malaysian Journal of Library \& Information Science, v. 12, n. 2, p. 35-55, 2007. 